\title{
Competing Complex Information Spreading in Multiplex Social Network
}

\author{
Xiang Li iD and Bocheng Hou \\ Yellow River Conservancy Technical Institute, Kaifeng, Henan Province 475004, China \\ Correspondence should be addressed to Bocheng Hou; houbocheng@yrcti.edu.cn
}

Received 5 March 2021; Accepted 28 April 2021; Published 11 May 2021

Academic Editor: Wei Wang

Copyright ( $) 2021$ Xiang Li and Bocheng Hou. This is an open access article distributed under the Creative Commons Attribution License, which permits unrestricted use, distribution, and reproduction in any medium, provided the original work is properly cited.

\begin{abstract}
Coevolution spreading dynamics on complex networks is a hot topic, which attracts much attention in network science. This paper proposes a mathematical model to describe the two competing complex information spreading dynamics on multiplex networks. An individual can only accept one of the two pieces of information. A heterogeneous mean-field theory is developed to describe the spreading dynamics. We reveal different regions through Monte Carlo simulations of the competing complex information spreading dynamics: no global information, one information dominant, and two information coexistence. We finally find that the heterogeneity of the multiplex networks' degree distributions does not qualitatively affect the results.
\end{abstract}

\section{Introduction}

Social networks, e.g., WeChat, Twitter, and Facebook, are the convenient way to express and share information with friends [1-5]. Students may retransmit an article to support or oppose the opinions of the authors. To describe the interaction of two types of information spreading dynamics on social networks, researchers used the framework of coevolution spreading dynamics on complex networks [6]. Generally speaking, scholars used complex networks to describe the social network, in which the nodes represent the individuals and edges stand for the relationships among individuals. Extensive empirical analyses revealed that social network exhibits degree heterogeneity, high clustering, multilayer, and temporality [7-18]. Including some of those characteristics, many research studies have been designed (see some important reviews in this field, e.g., reference [19-21]).

Based on the shared information's content, we can classify the information spreading dynamics into simple and complex information [22-24]. The simple information means that a single contact between the informed and susceptible individuals can trigger the infection transmission and widely used to describe epidemic spreading and simple news diffusion. In contrast, single contact cannot eliminate some complex information's legality and reliability (e.g., political networks). Therefore, social reinforcement is included in the dynamics of complex information spreading. In the following content, we present the progress for simple and complex information spreading detailedly.

The study about the simple information spreading on complex networks independently is the most widely investigated and revealed some critical results. For instance, simple information can always spread in the social network regardless of the values of information transmission probability when the heterogeneity of degree distribution is extremely strong [25-27]. Besides, the high clustering promotes the breakout of the information while suppresses the spreading size for large transmission probability [28]. Once several information are transmitted simultaneously, scholars revealed more novel phenomena. When two pieces of information are spreading on networks consecutively, the second information's outbreak threshold is larger than the threshold of the first information [29]. Karrer and Newman [30] further considered two competing spreading dynamics on the single network simultaneously. By using the competing percolation theory, they found that two epidemic cannot coexist in the thermodynamic limit (i.e., when the 
network size is extremely large). Sahneh and Scoglio [31] investigated two competing information on multiplex networks and computed the absolute-dominance and coexistence regions. When two information collaborate, i.e., if the acceptance probability for another information is enlarged when an individual accepts one information, the system exhibits a discontinuous phase transition [32-34]. Scholars considered another situation, asymmetric interacting, i.e., one spreading dynamic promotes the other. In contrast, the other one suppresses the spreading and reveals that the global epidemic will be greatly suppressed once the information spreading is included [35-37].

To include the social reinforcement in the dynamics of complex information spreading, the linear threshold model is the most widely used, in which an individual accepts the information only when the received information is larger than a threshold [22, 38-40]. Setting the final spreading size as the order parameter, researchers revealed the discontinuous phase transition in the system. More detailedly, the final spreading size versus the transmission probability exhibits a discontinuous growth. Liu et al. [41] investigated two complex information spreading consecutively on single complex network and revealed a transition for continuous and discontinuous phase transitions. Furthermore, Liu et al. [42] studied two complex synergetic information spreading dynamics on multiplex networks and revealed that the synergetic induced the discontinuous phase transition. To the best of our knowledge, there is still lacking a mathematical model to investigate two competing complex information spreading dynamics on multiplex networks. We will propose a mathematical model in section 2 and then develop a heterogeneous mean-field theory in section 3 . We further study the complex competing information spreading model by using Monte Carlo simulation in section 4. Finally, we conclude in section 5 .

\section{Competing Complex Information Spreading Model}

This section proposes the competing information spreading model on social network to describe different opinions evolving in the campus.

2.1. Network Description. Students usually discuss the events happened and diffuse the information or opinions through social platforms. We here use a multiplex network $G=\left(N_{A}, N_{B}, E_{A}, E_{B}\right)$ to describe the multiplex social network with two layers $A$ and $B$, where $N_{A}$ and $N_{B}$, respectively, represent the sizes of networks $A$ and $B$ and $E_{A}$ and $E_{B}$, respectively, stand for the number of edges in of networks $A$ and $B$. Nodes stand for the student users, and edges mean the relationships among those users. If two students $i$ and $j$ communicate with each other in network $A$ $(B)$, an edge exists between them.

We assume there are $n=\left|N_{A}\right|=\left|N_{B}\right|$ students in the social network and $e_{A}=\left|E_{A}\right|$ and $e_{B}=\left|E_{B}\right|$ connections among them. Therefore, the average degrees of the social network are $\widetilde{k_{A}}=\left(2 e_{A} / n\right)$ and $\widetilde{k_{B}}=\left(2 e_{B} / n\right)$, respectively.
The two networks' degree distribution is denoted as $P_{A}\left(k_{A}\right)$ and $P_{B}\left(k_{B}\right)$, respectively. Nodes with large degrees mean that they have more communication with friends and always act as hubs. A node in two networks represents that it communicates with others through two platforms. To build the multiplex network, we first generate the intralayer edges using the uncorrelated configuration model proposed in reference [43] according the degree distributions $P_{A}\left(k_{A}\right)$ and $P_{B}\left(k_{B}\right)$. Then, we build the interlayer edges randomly, that is to say, we randomly match two nodes in the two subnetworks.

2.2. Competing Spreading Dynamics. In social networks, students always express different opinions on the same event, and as a result, additional information is competing with each other. In reality, adopting an idea is risky. Therefore, social reinforcement is included. Social reinforcement means that the adoption of the information requires multiple verifications from friends. We here adopt a generalized susceptible-informed-recovered model to describe the two information spreading dynamics. The susceptible node means that it does not know the information and can adopt the information. An informed node stands for that adopted the information and willing to transmit it to friends. A node in the recovered state means that it has lost interest in the information.

The two competing information spreading dynamics are denoted as $\ell_{A}$ and $\ell_{B}$. We assume information $\ell_{A}$ and $\ell_{B}$, respectively, spread on networks $A$ and $B$ simultaneously. The competing between two dynamics means that a node cannot adopt two information simultaneously. The two competing spreading dynamics evolve as follows. Initially, we randomly select a node infected by $\ell_{A}$ and $\ell_{B}$ in two subnetworks. At every time step, every node informed by information $\ell_{h}(h \in\{A, B\})$ will transmit the information to its neighboring nodes in network $h$ with probability $\lambda$. To eliminate the information's credibility, we include the social reinforcement and assume a node adopting information $\ell_{h}$ must receive $T>1$ pieces. A susceptible node $i$, respectively, obtains $m_{A} \geq T$ and $m_{B} \geq T$ pieces of $\ell_{A}$ and $\ell_{B}$ information at time step $t$, and it adopts information $\ell_{A}$ with probability $m_{A} /\left(m_{A}+m_{B}\right)$ and adopts information $\ell_{B}$ with probability $m_{B} /\left(m_{A}+m_{B}\right)$. The informed nodes are recovered with probability $\gamma$. The two competing spreading dynamics evolve until there are no nodes in the informed state. The pseudocode of the competing spreading dynamics as shown in Algorithm 1. At each time step, the time complexity of the algorithm is $\mathcal{O}(E)$, where $E$ is the number of edges in the network. The space complexity is $\mathcal{O}(N)$.

\section{Theoretical Analysis}

Mathematically, the competing information spreading dynamics on social networks can be described by using the heterogeneous mean-field approach $[25,26,44]$, which is widely used in investigating the dynamics on networks. There are some basic assumptions behind the heterogeneous mean-field approach as follows: 
(1) Input: network $G$, and dynamical parameters $\lambda, T, \gamma, w$;

(2) Output: spreading sizes of information $\ell_{A}$ and $\ell_{B}$ and denoted as $R_{A}$ and $R_{B}$;

(3) $t \leftarrow 0$;

(4) Randomly a seed for information $\ell_{A}$ and $\ell_{B}$, and put them into queue $Q_{A}$;

(5) while $Q_{A}$ is not empty do

(6) $m_{A} \leftarrow 0$;

(7) $m_{B} \leftarrow 0$;

(8) Initialize $Q_{B}$ to be empty;

(9) $\delta t \leftarrow 1 /$ length $\left(Q_{A}\right)$;

(10) for $i=1$ to length $\left(Q_{A}\right)$ do

(11) if Node $i$ adopt the information $\ell_{A}$ then Node $i$ transmits the information to every susceptible neighbor $j$ in subnetwork $A$ with probability $\lambda$;

(12) if Node $j$ received the information $\ell_{A}$ successfully then

(13) $\quad m_{A} \leftarrow m_{A}+1$;

(14) end if

(15) end if

(16) if Node $i$ adopt the information $\ell_{B}$ then

(17) Node $i$ transmits the information to every susceptible neighbor in subnetwork $B$ with probability $\lambda$;

(18) if Node $j$ received the information $\ell_{A}$ successfully then

$m_{A} \leftarrow m_{A}+1$

end if

if $m_{A} \geq T$ and $m_{B} \geq T$ then

Node $j$ adopts information $\ell_{A}$ with probability $m_{A} /\left(m_{A}+m_{B}\right)$;

if Node $j$ adopts information $\ell_{A}$ then

Adding node $j$ into queue $Q_{B}$;

end if

Node $j$ adopts information $\ell_{B}$ with probability $m_{B} /\left(m_{A}+m_{B}\right)$;

if Node $j$ adopts information $\ell_{B}$ then

Adding node $j$ into queue $Q_{B}$;

end if

end if

if $m_{A} \geq T$ and $m_{B}<T$ then

Node $j$ adopts information $\ell_{A}$ with probability 1 ;

Adding node $j$ into queue $Q_{B}$;

end if

if $m_{A}<T$ and $m_{B} \geq T$ then

Node $j$ adopts information $\ell_{B}$ with probability 1;

Adding node $j$ into queue $Q_{B}$;

end if

end for

for $i=1$ to length $\left(Q_{A}\right)$ do

Recovering node $i$ with probability $\gamma$;

if Node $i$ recovers then

Delete node $i$ from queue $Q_{A}$;

end if

end for

for $i=1$ to length $\left(Q_{B}\right)$ do

Adding node $i$ to queue $Q_{A}$;

Deleting node $i$ from queue $Q_{B}$;

end for

end while 
(i) The network size is large enough.

(ii) There are no degree-degree correlations in the network.

(iii) The infection probability of informed nodes is independent, i.e., there are no dynamical correlations among nodes. Based on the above assumptions, we derive the evolution equations for competing information spreading dynamics.

To describe the evolution equations, we use the following mathematical notations $s_{k_{A}}^{\ell_{A}}(t), \rho_{k_{A}}^{\ell_{A}}(t)$, and $r_{k_{A}}^{\ell_{A}}(t)$ to represent the probability that a node with degree $k_{A}$ in subnetwork $A$ is in the susceptible, informed, and recovered state for information $\ell_{A}$ at time $t$. Similarly, we use $s_{k_{B}}^{\ell_{B}}(t)$, $\rho_{k_{B}}^{\ell_{B}}(t)$, and $r_{k_{B}}^{\ell_{B}}(t)$ to represent the probability that a node with degree $k_{B}$ in subnetwork $B$ is in the susceptible, informed, and recovered state for information $\ell_{B}$ at time $t$. Since the symmetry of the two spreading dynamics, we only describe the evolution equations for one of them. At time step $t$, the fraction of nodes in susceptible, informed, and recovered for information $\ell_{h} \quad(h \in\{A, B\})$ is $S^{\ell_{h}}(t)=\sum P_{h}\left(k_{h}\right) s_{k_{h}}^{\ell_{h}}(t), \quad \rho^{\ell_{h}}(t)=\sum P_{h}\left(k_{h}\right) \rho_{k_{h}}^{\ell_{h}}(t), \quad$ and $R^{\ell_{h}}(t)=\sum P_{h}\left(k_{h}\right) r_{k}^{t_{h}}(t)$, respectively. In the following, we derive the evolutions of $s_{k_{h}}^{\ell_{h}}(t), \rho_{k_{h}}^{\ell_{h}}(t)$, and $r_{k_{h}}^{\ell_{h}}(t)$.

The fraction of $s_{k_{h}}^{\ell_{h}}(t)$ decreases once node $i$ with degree $k k_{h}$ in subnetwork $h$ becomes informed. On the one hand, node $i$ must receive $m_{\ell_{h}} \geq T$ pieces of information $\ell_{h}$ in subnetwork $h$ at time $t$. In uncorrelated network, the probability of node $i$ connecting to an informed node $j$ with degree $k_{h}^{\prime}$ is $k_{h}^{\prime} P_{h}\left(k_{h}\right) /\left\langle k_{h}\right\rangle$. Therefore, the probability of node $j$ transmits the information to node $i$ successfully which is $\lambda k_{h}^{\prime} P_{h}\left(k_{h}\right) /\left\langle k_{h}\right\rangle \rho_{k_{h}}^{\ell_{h}}(t)$. The pieces of information $\ell_{h}$ that node $i$ received at time $t$ are

$$
m_{\ell_{h}}=k_{h} \lambda \frac{1}{\left\langle k_{h}\right\rangle} \sum_{k_{h}^{\prime}} k_{h}^{\prime} P_{h}\left(k_{h}\right) \rho_{k_{h}}^{\ell_{h}}(t) .
$$

According to descriptions of the model, we know node $i$ adopts information $\ell_{h}$ with probability

$$
\xi_{\ell_{h}}=\frac{m_{\ell_{h}} \delta_{m_{\ell_{h}} \geq T}}{m_{\ell_{h}} \delta_{m_{\ell_{h}} \geq T}+m_{\ell_{g}} \delta_{m_{\ell_{g}} \geq T}},
$$

where $\delta_{m_{\ell_{h}} \geq T}=1$ if $m_{\ell_{h}} \geq T$; otherwise, $m_{\ell_{h}} \geq T=0$. Note that $\ell_{g}$ is the other type of information. Based the above discussions, we know the evolution of $s_{k}^{\ell_{h}}(t)$ as

$$
\frac{\mathrm{d} s_{k_{h}}^{\ell_{h}}(t)}{\mathrm{d} t}=-s_{k_{h}}^{\ell_{h}}(t) s_{k_{h}}^{\ell_{g}}(t) \xi_{\ell_{h}} .
$$

With a similar discussion, we know the evolutions of $\rho_{k_{h}}^{\ell_{h}}(t)$ and $r_{k_{h}}^{\ell_{h}}(t)$ as

$$
\begin{aligned}
& \frac{\mathrm{d} \rho_{k_{h}}^{\ell_{h}}(t)}{\mathrm{d} t}=-\gamma \rho_{k_{h}}^{\ell_{h}}(t)+s_{k_{h}}^{\ell_{h}}(t) s_{k_{h}}^{\ell_{g}}(t) \xi_{\ell_{h}}, \\
& \frac{\mathrm{d} \rho_{k_{h}}^{\ell_{h}}(t)}{\mathrm{d} t}=\gamma \rho_{k_{h}}^{\ell_{h}}(t),
\end{aligned}
$$

respectively.

\section{Results Analysis}

In this section, we numerically study the competing information spreading on multiplex networks detailedly. We study the competing information spreading on two types of multiplex networks. The first one is ER-ER multiplex network, i.e., networks $A$ and $B$ are Erdös-Rényi (ER), in which the degree distribution follows a Poisson distribution. That is to say, the degree distribution of subnetwork $h \in\{A, B\}$ is $P_{h}\left(k_{h}\right)=\left(\left\langle k_{h}\right\rangle^{k_{h}} / k_{h} !\right) e^{-\left\langle k_{h}\right\rangle}$, where $\left\langle k_{h}\right\rangle$ is the average degree of subnetwork $h$. We assume there is no interlayer degree-degree correlations, and thus the joint degree distribution is $P\left(k_{A}, k_{B}\right)=\left(\left\langle k_{A}\right\rangle^{k_{A}} / k_{A} !\right) e^{-\left\langle k_{A}\right\rangle}\left(\left\langle k_{B}\right\rangle^{k_{B}}\right.$ $\left.k_{B} !\right) e^{-\left\langle k_{B}\right\rangle}$. For ER-ER multiplex networks, the degree distribution is homogeneous. In reality, many real-data revealed that the degree distribution is heterogeneous. We therefore use the SF-SF multiplex networks, in which the two subnetworks are both scale-free networks. The degree distribution of subnetwork $h \in\{A, B\}$ is $P_{h}\left(k_{h}\right)=\left(1 / \varepsilon_{h}\right) k_{h}^{-\gamma_{h}}$, where $\varepsilon_{h}=\sum k_{h} k_{h}^{-\gamma_{h}}$. Similar with ER-ER multiplex networks, we assume there is no interlayer degree-degree correlations, and the joint degree distribution is $P\left(k_{A}, k_{B}\right)=$ $\left(1 / \varepsilon_{A} \varepsilon_{B}\right) k_{A}^{-\gamma_{A}} k_{B}^{-\gamma_{B}}$. In numerical simulations, we set the network size $n=N_{A}=N_{B}=2 \times 10^{4}$ and $\left\langle k_{A}\right\rangle=\left\langle k_{B}\right\rangle=10$. The results presented in the paper are averaged over at least 1000 times.

4.1. ER-ER Multiplex Networks. Because the social reinforcement is included in the information spreading dynamics, we first study the effects of initial seed size in Figure 1. To study the fraction of new informed nodes by each information, we investigate $R_{A}-\rho_{0}$ and $R_{B}-\rho_{0}$ versus $\lambda_{A}$ for different values of $\lambda_{B}$ and $\rho_{0}$. For small values of $\lambda_{B}$, e.g., $\lambda_{B}=0.2$, the fraction of new informed nodes by the seeds depends on the fraction of seeds shown in Figure 1(a). When the seed is small (e.g., $\rho_{0}=0.01$ ), any values of information transmission probability $\lambda_{A}$ cannot trigger the global outbreak for information $\ell_{A}$. Since the social reinforcement is included, a small fraction of seeds cannot further adopt the information. With the increase in $\rho_{0}$, the global outbreak of $\ell_{A}$ becomes possible when $\lambda_{A}$ is above the threshold point $\lambda_{A}^{c}$. Note that $\lambda_{A}^{c}$ can be numerically determined by studying the variability $\chi$ of $R_{A}-\rho_{0}$, and at the threshold point, $\chi$ exhibits a peak. We do not show $\chi$ in this paper. Note that the global information of $\ell_{B}$ cannot outbreak when $\lambda_{B}=0.2$, as shown in Figure $1(\mathrm{~b})$. For larger values of $\lambda_{B}$, such as $\lambda_{B}=0.5$ and 0.8 , we find that a small fraction of seeds cannot trigger the global outbreak for the two types of information (see Figures $1(\mathrm{c})-1(\mathrm{f}))$. For larger values of $\rho_{0}=0.05$ and 0.1 , $R_{A}-\rho_{0}$ increases with $\lambda_{A}$, meanwhile $R_{B}-\rho_{0}$ decreases with $\lambda_{A}$. We note that two types of information may coexist, and the coexistence conditions are discussed detailedly in Figure 2.

In Figure 2, we investigate the fractions of new informed nodes by the two types of information triggered by the initial seeds on a plane $\left(\lambda_{A}, \lambda_{B}\right)$. As shown in Figure 2(a), the global information can not trigger small values $\lambda_{A}$. Previous studies 

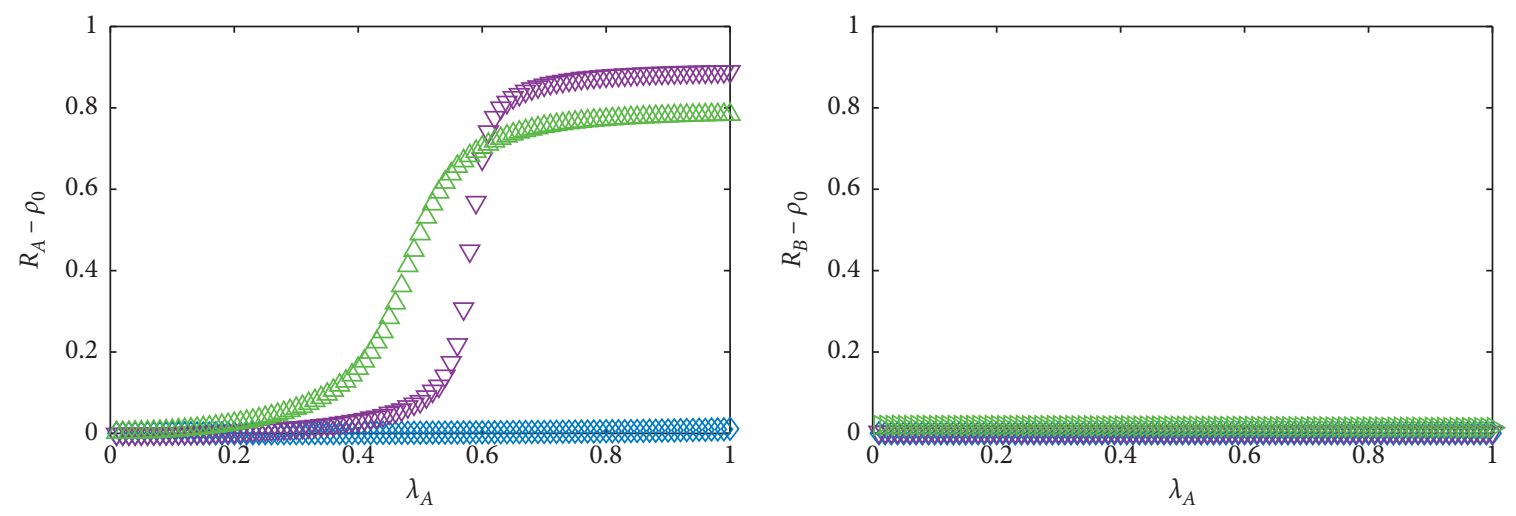

$\diamond \lambda_{B}=0.2, \rho_{0}=0.01$
$\nabla \lambda_{B}=0.2, \rho_{0}=0.05$
$\lambda_{B}=0.2, \rho_{0}=0.1$

$$
\begin{aligned}
& \diamond \lambda_{B}=0.2, \rho_{0}=0.01 \\
& \nabla \lambda_{B}=0.2, \rho_{0}=0.05 \\
& \triangleright \lambda_{B}=0.2, \rho_{0}=0.1
\end{aligned}
$$

(a)
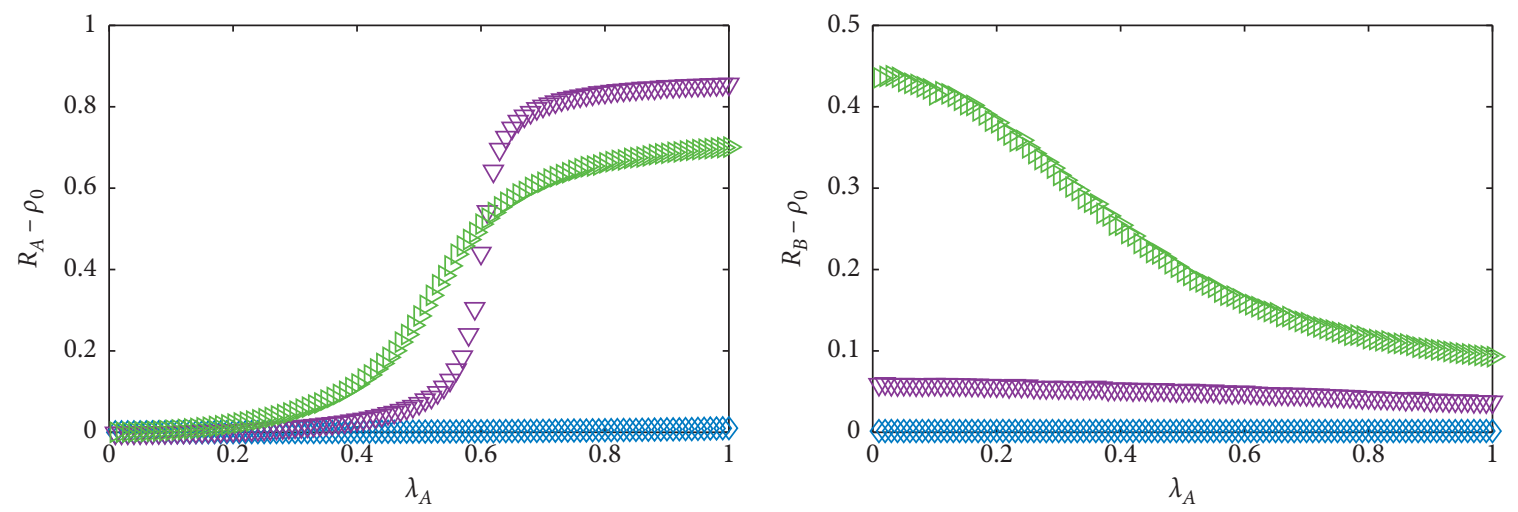

$$
\begin{aligned}
& \diamond \lambda_{B}=0.5, \rho_{0}=0.01 \\
& \nabla \lambda_{B}=0.5, \rho_{0}=0.05 \\
& \triangleright \lambda_{B}=0.5, \rho_{0}=0.1
\end{aligned}
$$

$$
\begin{aligned}
& \diamond \lambda_{B}=0.5, \rho_{0}=0.01 \\
& \nabla \lambda_{B}=0.5, \rho_{0}=0.05 \\
& \triangleright \lambda_{B}=0.5, \rho_{0}=0.1
\end{aligned}
$$

(d)
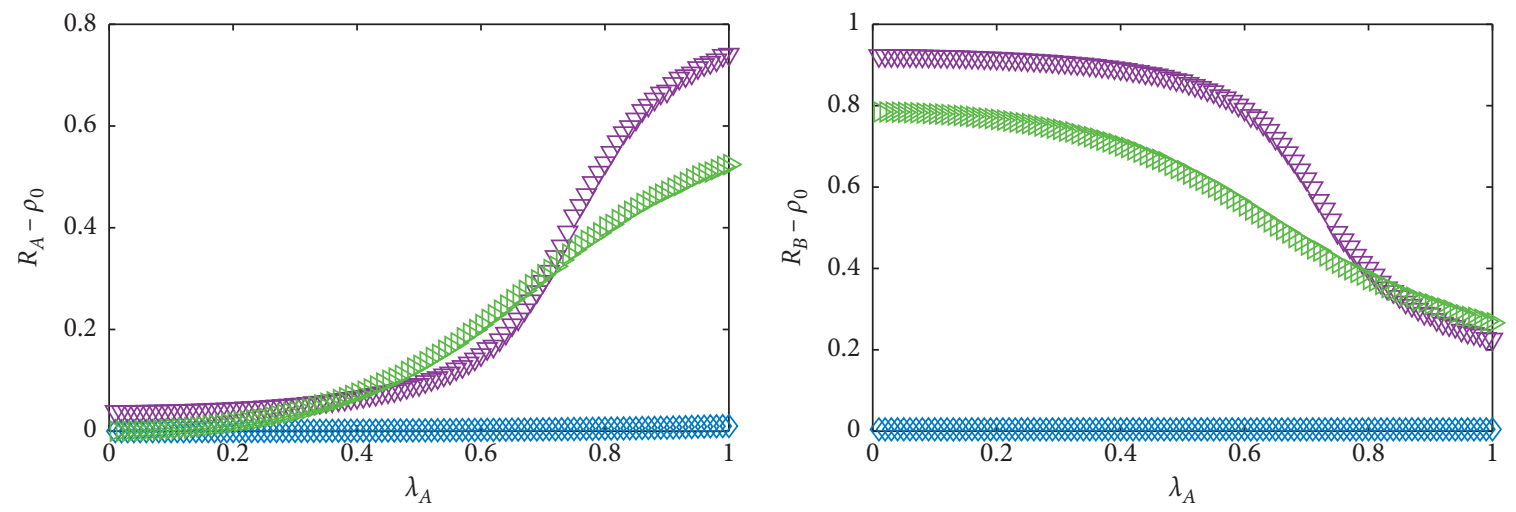

$$
\begin{aligned}
& \diamond \lambda_{B}=0.8, \rho_{0}=0.01 \\
& \nabla \lambda_{B}=0.8, \rho_{0}=0.05 \\
& \triangleright \lambda_{B}=0.8, \rho_{0}=0.1
\end{aligned}
$$

$$
\begin{aligned}
& \diamond \lambda_{B}=0.8, \rho_{0}=0.01 \\
& \nabla \lambda_{B}=0.8, \rho_{0}=0.05 \\
& \triangleright \lambda_{B}=0.8, \rho_{0}=0.1
\end{aligned}
$$

(e)

FIGURE 1: Competing information spreading on ER-ER multiplex networks: (a) The new informed information spreading size $R_{A}-\rho_{0}$ of information $\ell_{A}$; (b) new informed information spreading size $R_{B}-\rho_{0}$ of information $\ell_{B}$ as a function of $\lambda_{A}$ with $\lambda_{B}=0.2$; (c) $R_{A}-\rho_{0}$; (d) $R_{B}-\rho_{0} \lambda_{A}$ with $\lambda_{B}=0.5$; (e) $R_{A}-\rho_{0}$; (f) $R_{B}-\rho_{0} \lambda_{A}$ with $\lambda_{B}=0.8$. We set the threshold $T=2$, the average degrees of the two networks are $\left\langle k_{A}\right\rangle=\left\langle k_{B}\right\rangle=10$, and we set the recovery probability $\gamma=0.5$. 


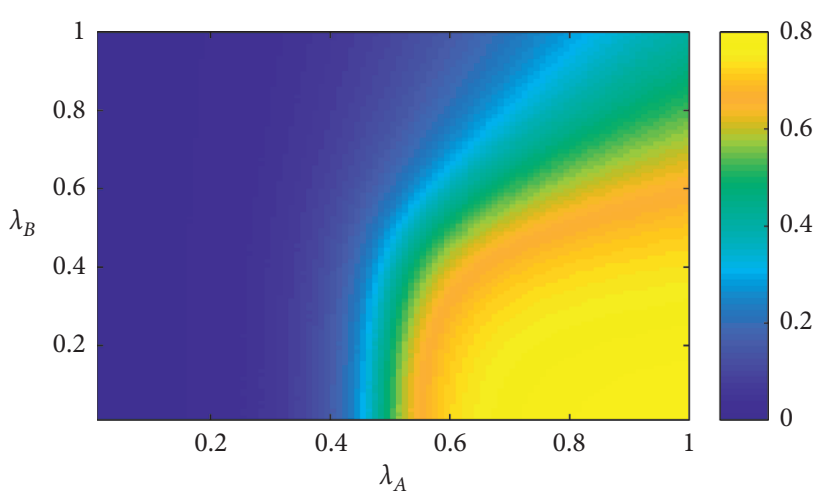

(a)

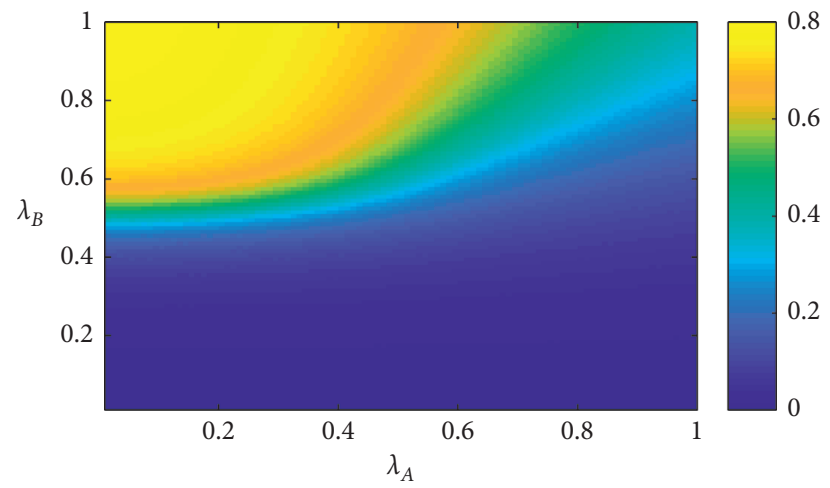

(b)

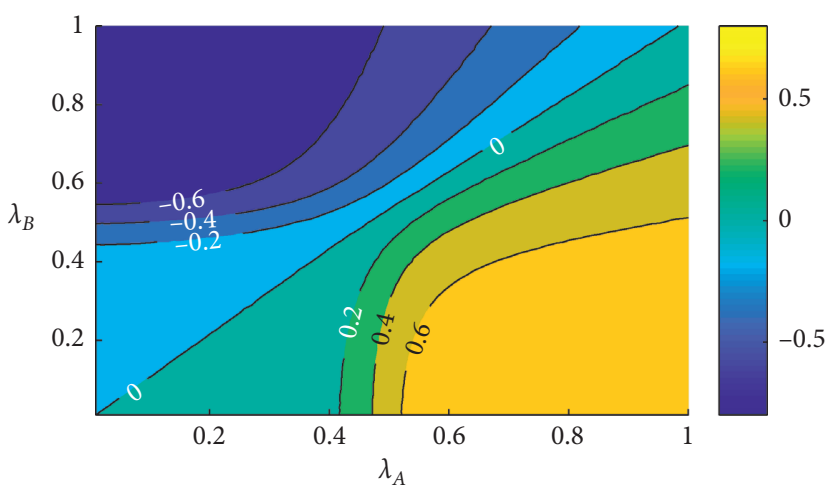

(c)

FIgURE 2: Competing information spreading on ER-ER multiplex networks versus information transmission probability: (a) the new informed information spreading size $R_{A}-\rho_{0}$ of information $\ell_{A}$; (b) new informed information spreading size $R_{B}-\rho_{0}$ of information $\ell_{B}$; (c) $R_{A}-R_{A}$ as a function of $\left(\lambda_{A}, \lambda_{B}\right)$. We set the threshold $T=2$, the average degrees of the two networks are $\left\langle k_{A}\right\rangle=\left\langle k_{B}\right\rangle=10$, and we set the initial fraction seed of the two types of information as $\rho_{0}=0.1$ and $\gamma=0.5$.

indicated that the global information outbreak threshold of information $\ell_{A}$ is $\lambda_{A}^{0}=1 /\left\langle k_{A}\right\rangle=0.1$ when there is only information $\ell_{A}$ spreading on network $A$. However, when the two competing information spreads on multiplex networks simultaneously, the global information cannot break when $\lambda_{A}$ slightly is more extensive than $\lambda_{A}^{0}$. We know that the competing information suppresses the spreading dynamics, and the global information outbreak threshold $\lambda_{A}^{c}$ is much larger than $\lambda_{A}^{0}$. With the two information spreading dynamics' symmetry mechanisms, we find the similar phenomena of $R_{B}-\rho_{0}$ in Figure 2(b).

One important question is the domination and coexistence of regions. Domination means that one information spreads to most nodes, while the other only transmits to a few nodes. Coexistence stands for the two types of information spread to a large fraction of nodes. To this end, we study $R_{A}-R_{B}$ in Figure 2(c). For the case of $R_{A}-R_{B}>0$, we know information $\ell_{A}$ dominates. When $R_{A}<R_{B}$, the information $\ell_{B}$ dominates. When $R_{A} \approx R_{B}$, the two types of information compete for the nodes. Combing Figures 2(a)2(c), we speculate information $\ell_{A}$ dominates when $\lambda_{A}>\lambda_{A}^{0}$ and $\lambda_{A} \gg \lambda_{B}$. Similarly, $\ell_{B}$ dominates when $\lambda_{B}>\lambda_{B}^{0}=1 /\langle k\rangle$ and $\lambda_{B} \gg \lambda_{A}$. The two types of information are competing with the nodes and can coexist when $\lambda_{A} \approx \lambda_{B}, \lambda_{A}>\lambda_{A}^{0}$, and $\lambda_{B}>\lambda_{B}^{0}$.
In Figure 3, we further study the effects of the average degrees on the competing information spreading dynamics when $\lambda_{A}=\lambda_{B}$. When the two types of information spreading on the two networks with the same average degree, i.e., $\left\langle k_{A}\right\rangle=\left\langle k_{B}\right\rangle=10$, we find that $R_{A}-\rho_{0}$ and $R_{B}-\rho_{0}$ exhibit the similar behavior, that is to say, $R_{A}-\rho_{0}$ and $R_{B}-\rho_{0}$ increase monotonously with the increase in $\lambda_{A}=\lambda_{B}$. However, $R_{A}-\rho_{0}$ first increases with $\lambda_{A}=\lambda_{B}$ and then decreases when $\left.\left\langle k_{A}\right\rangle\right\rangle\left\langle k_{B}\right\rangle$, e.g., $\left\langle k_{A}\right\rangle=15$ and $\left\langle k_{A}\right\rangle=10$. That is to say, there exists a peak at which $R_{A}-\rho_{0}$ reaches its maximum. Meanwhile, $R_{B}-\rho_{0}$ always increases with $\lambda_{A}=$ $\lambda_{B}$ regardless the values of $\left\langle k_{A}\right\rangle$ and $\left\langle k_{B}\right\rangle$.

4.2. SF-SF Multiplex Networks. Finally, we study the two competing information spreading on SF-SF multiplex networks in Figure 4 and investigate the effects of degree heterogeneity of the two networks on the spreading dynamics. Compared with the ER-ER networks, the SF-SF networks promote the spreading dynamics since the existence of some nodes with large degrees. In addition, we find that the degree heterogeneity does not qualitatively affect the phenomena presented in Figure 2. That is to say, the domination and coexistence regions are the same. The domination region of information $\ell_{A}$ is $\lambda_{A}>\lambda_{A}^{0}$ and $\lambda_{A} \gg \lambda_{B}$. Similarly, information 


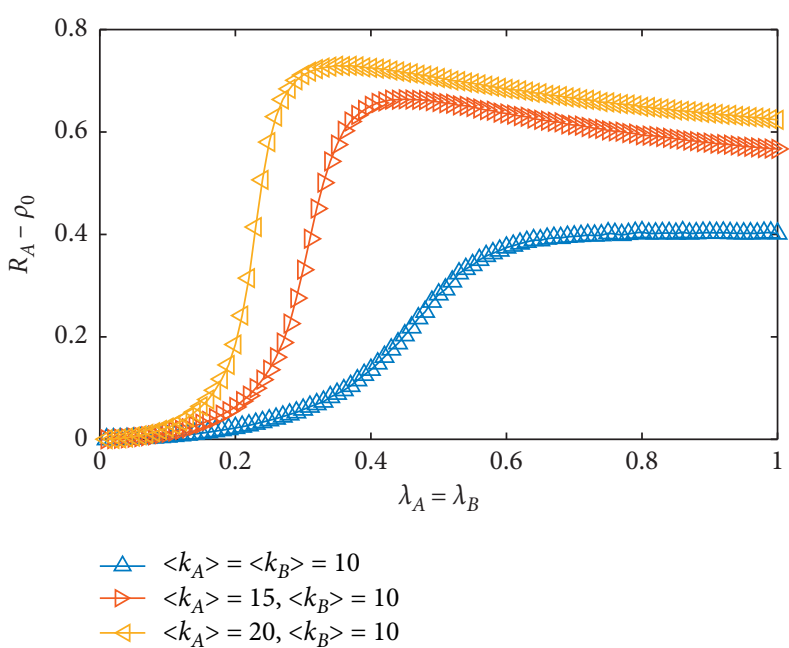

(a)

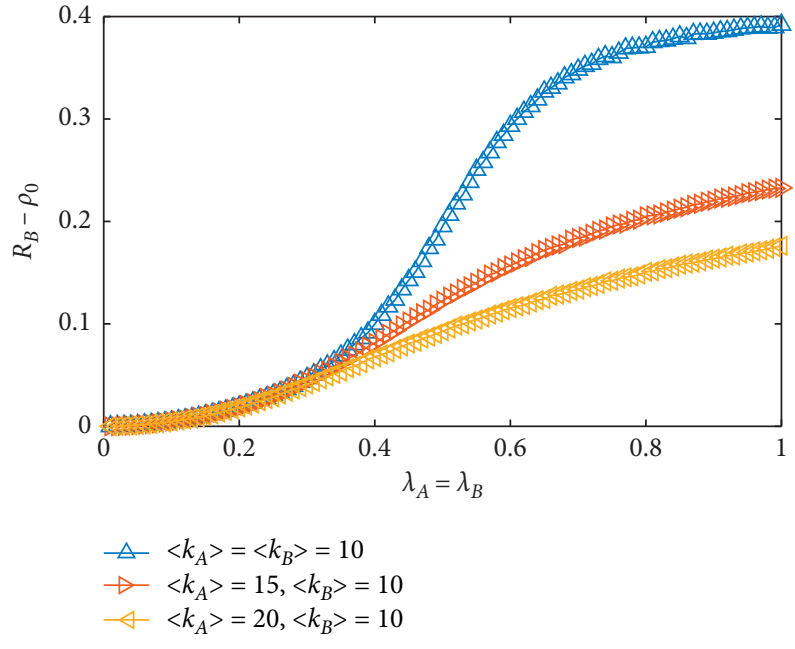

(b)

Figure 3: Competing information spreading on ER-ER multiplex networks: (a) the new informed information spreading size $R_{A}-\rho_{0}$ of information $\ell_{A}$; (b) new informed information spreading size $R_{B}-\rho_{0}$ of information $\ell_{B}$ function of $\lambda_{A}=\lambda_{B}$. We set the threshold $T=2$ and the initial fraction seed of the two types of information as $\rho_{0}=0.1$ and $\gamma=0.5$.

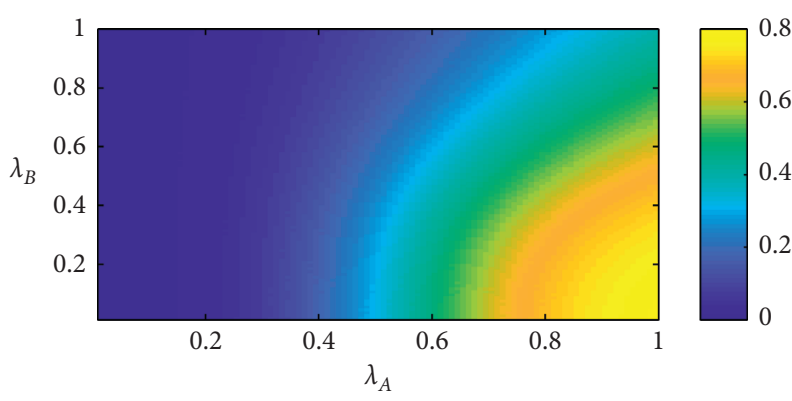

(a)

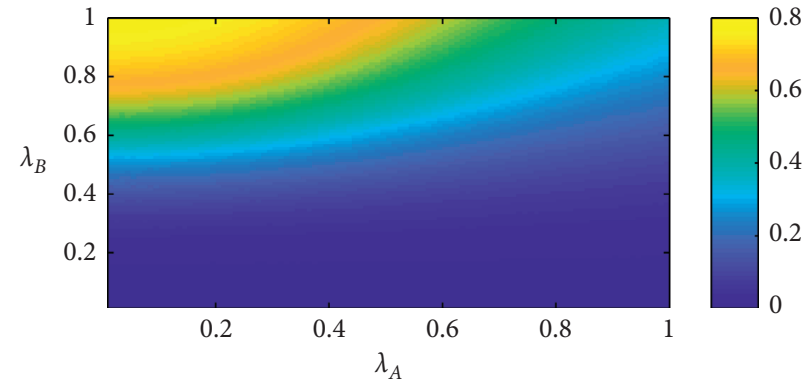

(b)

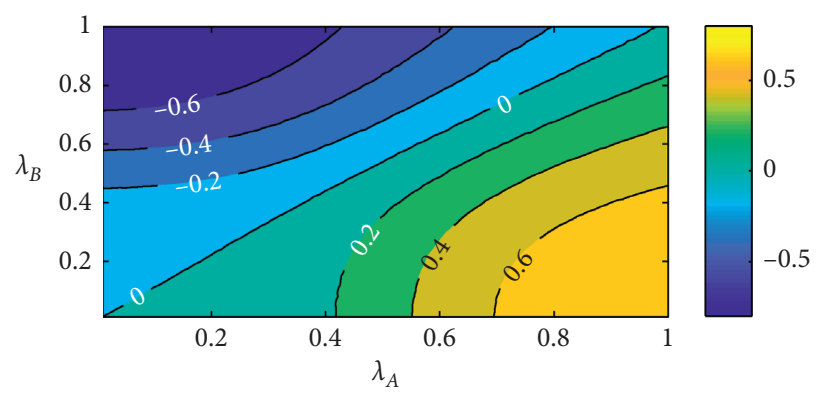

(c)

Figure 4: Competing information spreading on SF-SF multiplex networks versus information transmission probability: (a) the new informed information spreading size $R_{A}-\rho_{0}$ of information $\ell_{A}$; (b) new informed information spreading size $R_{B}-\rho_{0}$ of information $\ell_{B}$; (c) $R_{A}-R_{A}$ as a function of $\left(\lambda_{A}, \lambda_{B}\right)$. We set the threshold $T=2$, the average degrees of the two networks are $\left\langle k_{A}\right\rangle=\left\langle k_{B}\right\rangle=10$, the degree exponents are $\gamma_{A}=\gamma_{B}=3.0$, and we set the initial fraction seed of the two types of information as $\rho_{0}=0.1$ and $\gamma=0.5$.

$\ell_{B}$ domination region is $\lambda_{B}>\lambda_{B}^{0}=1 /\langle k\rangle$ and $\lambda_{B} \gg \lambda_{A}$. The coexistence region is $\lambda_{A} \approx \lambda_{B}, \lambda_{A}>\lambda_{A}^{0}$, and $\lambda_{B}>\lambda_{B}^{0}$.

\section{Discussion}

Coevolution spreading dynamics aims at describing interacting dynamics and revealing the phenomena induced by distinct interacting mechanisms. In this paper, we proposed a competing complex information model to describe two types of information diffusion on multiplex networks. A heterogeneous mean-field theory is developed to describe the model. Through extensive numerical simulations, we revealed the conditions of different regions. Specifically, no global information outbreak region is $\lambda_{A} \leq \lambda_{A}^{0}$ and $\lambda_{B} \leq \lambda_{B}^{0}$; 
information $\ell_{A}$ dominant is $\lambda_{A}>\lambda_{A}^{0}$ and $\lambda_{A} \gg \lambda_{B}$; information $\ell_{B}$ dominant is $\lambda_{B}>\lambda_{B}^{0}$ and $\lambda_{B} \gg \lambda_{A}$; and two information coexistence region is $\lambda_{A} \approx \lambda_{B}, \lambda_{A}>\lambda_{A}^{0}$, and $\lambda_{B}>\lambda_{B}^{0}$. When the two competing complex information spread on a multiplex with heterogeneous degree distribution, the phenomena do not qualitatively be affected. The results presented in this paper may shed some light into further investigating the competing spreading, such as design of effective strategy to make one spreading always win regardless of the other spreading dynamics and network structures.

\section{Data Availability}

The data used to support the findings of this study are available from the corresponding author upon request.

\section{Conflicts of Interest}

The authors declare that they have no conflicts of interest.

\section{Acknowledgments}

This work was partially supported by the Ministry of Education Science and Technology Development Center University Research Innovation Fund: "Qingtai Digital Intelligence Integration" Collaborative Innovation, no. 2020 QT20.

\section{References}

[1] D. Knoke and S. Yang, Social Network Analysis, Sage Publications, Thousand Oaks, CF, USA, 2019.

[2] G. Kossinets and D. J. Watts, "Empirical analysis of an evolving social network," Science, vol. 311, no. 5757, pp. 88-90, 2006.

[3] P. J. Carrington, J. Scott, and S. Wasserman, Models and Methods in Social Network Analysis, Vol. 28, Cambridge University Press, Cambridge, UK, 2005.

[4] Y. Amichai-Hamburger and G. Vinitzky, "Social network use and personality," Computers in Human Behavior, vol. 26, no. 6, pp. 1289-1295, 2010.

[5] M. Burke, C. Marlow, and T. Lento, "Social network activity and social well-being," in Proceedings of the SIGCHI Conference on Human Factors in Computing Systems, pp. 19091912, Atlanta, GO, USA, April 2010.

[6] W. Wang, Q.-H. Liu, J. Liang, Y. Hu, and T. Zhou, "Coevolution spreading in complex networks," Physics Reports, vol. 820, pp. 1-51, 2019.

[7] M. Newman, Networks, Oxford University Press, Oxford, UK, 2018.

[8] A.-L. Barabási, "The new science of networks," Physics Today, vol. 6, no. 5, 2003.

[9] A.-L. Barabási and R. Albert, "Emergence of scaling in random networks," Science, vol. 286, no. 5439, pp. 509-512, 1999.

[10] F. Papadopoulos, M. Kitsak, M. Á. Serrano, M. Boguñá, and D. Krioukov, "Popularity versus similarity in growing networks," Nature, vol. 489, no. 7417, pp. 537-540, 2012.

[11] P. Holme, "Rare and everywhere: perspectives on scale-free networks," Nature Communications, vol. 10, p. 1016, 2019.
[12] A. Clauset, C. R. Shalizi, and M. E. J. Newman, "Power-law distributions in empirical data," SIAM Review, vol. 51, no. 4, pp. 661-703, 2009.

[13] M. Kivelä, A. Arenas, M. Barthelemy, J. P. Gleeson, Y. Moreno, and M. A. Porter, "Multilayer networks," Journal of Complex Networks, vol. 2, no. 3, pp. 203-271, 2014.

[14] M. De Domenico, A. Solé-Ribalta, E. Cozzo et al., "Mathematical formulation of multilayer networks," Physical Review $X$, vol. 3, p. 041022, 2013.

[15] M. De Domenico, V. Nicosia, A. Arenas, and V. Latora, "Structural reducibility of multilayer networks," Nature Communications, vol. 6, p. 6864, 2015.

[16] P. Holme, "Modern temporal network theory: a colloquium," The European Physical Journal B, vol. 88, p. 234, 2015.

[17] P. Holme and J. Saramäki, Temporal Network Theory, Vol. 2, Springer, Berlin, Germany, 2019.

[18] X. Xue, L. Pan, M. Zheng, and W. Wang, "Network temporality can promote and suppress information spreading," Chaos: An Interdisciplinary Journal of Nonlinear Science, vol. 30, no. 11, p. 113136, 2020.

[19] Z.-K. Zhang, C. Liu, X.-X. Zhan, X. Lu, C.-X. Zhang, and Y.-C. Zhang, "Dynamics of information diffusion and its applications on complex networks," Physics Reports, vol. 651, pp. 1-34, 2016.

[20] C. Castellano, S. Fortunato, and V. Loreto, "Statistical physics of social dynamics," Reviews of Modern Physics, vol. 81, no. 2, pp. 591-646, 2009.

[21] M. De Domenico, C. Granell, M. A. Porter, and A. Arenas, "The physics of spreading processes in multilayer networks," Nature Physics, vol. 12, no. 10, pp. 901-906, 2016.

[22] D. J. Watts, "A simple model of global cascades on random networks," Proceedings of the National Academy of Sciences, vol. 99, no. 9, pp. 5766-5771, 2002.

[23] D. J. Watts and P. S. Dodds, "Influentials, networks, and public opinion formation," Journal of Consumer Research, vol. 34, no. 4, pp. 441-458, 2007.

[24] E. Bakshy, J. M. Hofman, W. A. Mason, and D. J. Watts, "Everyone's an influencer: quantifying influence on twitter," in Proceedings of the Fourth ACM International Conference on Web Search and Data Mining, pp. 65-74, Hong Kong, China, February 2011.

[25] R. Pastor-Satorras and A. Vespignani, "Epidemic dynamics and endemic states in complex networks," Physical Review E, vol. 63, p. 066117, 2001.

[26] R. Pastor-Satorras and A. Vespignani, "Epidemic dynamics in finite size scale-free networks," Physical Review E, vol. 65, p. 035108, 2002.

[27] S. Gómez, A. Arenas, J. Borge-Holthoefer, S. Meloni, and Y. Moreno, "Discrete-time Markov chain approach to contact-based disease spreading in complex networks," $E P L$ (Europhysics Letters), vol. 89, no. 3, p. 38009, 2010.

[28] M. E. Newman, "Random graphs with clustering," Physical Review Letters, vol. 103, p. 058701, 2009.

[29] M. E. Newman, "Threshold effects for two pathogens spreading on a network," Physical Review Letters, vol. 95, p. 108701, 2005.

[30] B. Karrer and M. E. Newman, "Competing epidemics on complex networks," Physical Review E, vol. 84, p. 036106, 2011.

[31] F. D. Sahneh and C. Scoglio, "Competitive epidemic spreading over arbitrary multilayer networks," Physical Review E, vol. 89, p. 062817, 2014.

[32] L. Chen, "Persistent spatial patterns of interacting contagions," Physical Review E, vol. 99, p. 022308, 2019. 
[33] L. Chen, F. Ghanbarnejad, and D. Brockmann, "Fundamental properties of cooperative contagion processes," New Journal of Physics, vol. 19, no. 10, p. 103041, 2017.

[34] W. Cai, L. Chen, F. Ghanbarnejad, and P. Grassberger, "Avalanche outbreaks emerging in cooperative contagions," Nature Physics, vol. 11, no. 11, pp. 936-940, 2015.

[35] W. Wang, M. Tang, H. Yang, Y. Do, Y.-C. Lai, and G. Lee, "Asymmetrically interacting spreading dynamics on complex layered networks," Scientific Reports, vol. 4, p. 5097, 2014.

[36] C. Granell, S. Gómez, and A. Arenas, "Dynamical interplay between awareness and epidemic spreading in multiplex networks," Physical Review Letters, vol. 111, p. 128701, 2013.

[37] W. Wang, Q.-H. Liu, S.-M. Cai, M. Tang, L. A. Braunstein, and H. E. Stanley, "Suppressing disease spreading by using information diffusion on multiplex networks," Scientific Reports, vol. 6, p. 29259, 2016.

[38] M. S. Granovetter, "The strength of weak ties," American Journal of Sociology, vol. 78, no. 6, pp. 1360-1380, 1973.

[39] J. P. Gleeson and D. J. Cahalane, "Seed size strongly affects cascades on random networks," Physical Review E, vol. 75, p. 056103, 2007.

[40] W. Wang, M. Tang, H.-F. Zhang, and Y.-C. Lai, "Dynamics of social contagions with memory of nonredundant information," Physical Review E, vol. 92, p. 012820, 2015.

[41] Q.-H. Liu, L.-F. Zhong, W. Wang, T. Zhou, and H. Eugene Stanley, "Interactive social contagions and co-infections on complex networks," Chaos, vol. 28, p. 013120, 2018.

[42] Q.-H. Liu, W. Wang, S.-M. Cai, M. Tang, and Y.-C. Lai, "Synergistic interactions promote behavior spreading and alter phase transitions on multiplex networks," Physical Review E, vol. 97, p. 022311, 2018.

[43] M. Catanzaro, M. Boguná, and R. Pastor-Satorras, "Diffusionannihilation processes in complex networks," Physical Review E, vol. 71, p. 027103, 2005.

[44] W. Wang, M. Tang, H. E. Stanley, and L. A. Braunstein, "Unification of theoretical approaches for epidemic spreading on complex networks," Reports on Progress in Physics, vol. 80, p. $036603,2017$. 